\title{
Os conhecimentos, atitudes e comportamentos sobre SIDA dos adolescentes portugueses do meio urbano e não-urbano*
}

\author{
KNOWLEDGE, ATTITUDES AND BEHAVIOURS ABOUT AIDS IN PORTUGUESE ADOLESCENTS IN \\ URBAN AND NON-URBAN ENVIRONMENT
}

\section{CONOCIMIENTOS, ACTITUDES Y COMPORTAMIENTOS SOBRE EL SIDA EN LOS ADOLESCENTES PORTUGUESES DEL MEDIO URBANO Y NO URBANO}

\section{Assunção Dores Laranjeira de Almeida ${ }^{1}$, Carlos Fernandes da Silva ${ }^{2}$, Gabriel Saraiva da Cunha ${ }^{3}$}

* Extraído da
dissertação "Os
adolescentes e o
HIVISIDA".
Faculdade de
Medicina,
Universidade de
Coimbra, 2003.
1Enfermeira
Especialista em
Saúde Infantil e
Pediátrica. Assistente
2. ${ }^{\circ}$ triênio da Escola
Superior de Saúde da
Universidade de
Aveiro. Doutoranda
em Ciências da
Saúde. Campus
Universitário de
Santiago, Portugal -
aalmeida@
esssua.ua.pt.
2 Professor
Catedrático da
Universidade de
Aveiro. Campus
Universitário de
Santiago, Portugal -
csilva@dce.ua.pt
3 Professor Agregado
da Faculdade de
Medicina da
Universidade de
Coimbra. Director do
serviço de Doenças
Infecciosas dos
Hospitais da
Universidade de
Coimbra. Hospitais
da Universidade de
Coimbra,Portugal -
saraiva@huc.min-
saude.pt

RESUMO

O principal objetivo desta investigação foi identificar e comparar os conhecimentos, atitudes e comportamentos preventivos sobre a Síndrome da Imunodeficiência Adquirida (SIDA) dos adolescentes que freqüentam escola secundária inserida em meio urbano com os adolescentes que freqüentam escola secundária inserida em meio não urbano. Para o efeito procedeu-se a um estudo descritivo numa amostra populacional de 826 adolescentes (455 do meio urbano e 371 do meio não-urbano). $\mathrm{O}$ instrumento de coleta de dados adotado foi efetuado com base no modelo conceptual Health Belief Model. Constatou-se haver relação entre o meio de inserção dos adolescentes e o conhecimento da SIDA enquanto ameaça grave; o conhecimento sobre os riscos de contágio da SIDA e as atitudes diante da SIDA.

\section{DESCRITORES}

Síndrome de Imunodeficiência Adquirida.

Adolescente.

Conhecimento.

Atitude.

Comportamento.

\section{ABSTRACT}

The main objective of this investigation was to identify and compare preventive knowledge, attitudes and behaviors regarding AIDS between adolescents at the secondary school in urban and non-urban areas in Portugal. This is a descriptive study with a sample of 826 individuals (455 adolescents from urban environment and 371 from a nonurban environment). The instrument for data collection adopted was based on the Health Belief Model conceptual model. It was recognized that there is a relation between the environment insertion of the adolescents and the recognition of AIDS as a serious threat, and the knowledge about the risks of infection with AIDS and the attitudes towards AIDS.

\section{KEY WORDS}

Acquired Immunodeficiency Syndrome.

Adolescent.

Knowledge.

Attitude.

Behavior.

\section{RESUMEN}

El principal objetivo de esta investigación fue identificar y comparar los conocimientos, actitudes y comportamientos preventivos sobre el SIDA (HIV Síndrome da Imunodeficiência Adquirida) en los adolescentes que frecuentan la escuela secundaria insertada en el medio urbano con los adolescentes que frecuentan la escuela secundaria en el medio no urbano. Para tal efecto se procedió un estudio descriptivo de una muestra poblacional de 826 adolescentes (455 del medio urbano y 371 del medio no urbano). El instrumento de la coleta de datos adoptado fue efectuado con base al modelo conceptual Health Belief Model. Fue constatado existir una relación entre el medio de inserción de los adolescentes y el conocimiento sobre el SIDA, según su amenaza grave; el conocimiento sobre los riesgos de contagio del SIDA y las actitudes hacia el SIDA.

\section{DESCRIPTORES}

Síndrome de Inmunodeficiência Adquirida.

Adolescente.

Conocimiento.

Actitud.

Conducta. 


\section{INTRODUÇÃO}

O trabalho apresentado foi efectuado em Portugal na região Centro do País. A 12 de Março de 2001 residiam em Portugal 10355824 indivíduos. Na região Centro existiam na mesma data 2303579 indivíduos dos quais 1100721 eram homens e 1202858 eram mulheres. O período intercensitário (entre o ano de 1991 e 2001) caracterizou-se por: um crescimento demográfico $(5 \%)$ que se deve quase exclusivamente aos fluxos migratórios; acentuou-se o envelhecimento demográfico; a relação de masculinidade aumentou em todas as regiões; a pílula foi o método contraceptivo utilizado maioritariamente; Portugal é o país com menor proporção de jovens comparado com a média europeia; em Portugal os jovens dos 15 aos 34 anos tendem a fazer a transição da vida de estudante para o mercado de trabalho cerca dos dezoito anos, mais cedo que nos países nórdicos, mas ao mesmo tempo que os italianos ${ }^{(1)}$.

O presente estudo incidiu sobre os conhecimentos, atitudes e comportamentos de saúde relativas à SIDA nos adolescentes inseridos em meio urbano e não urbano.

O meio urbano é aquele para o qual é reconhecida vocação para o processo de urbanização e de edificaçãa ${ }^{(2)}$.

Atendendo às definições de meios rurais utilizados nos diversos Estados Membros da União Europeia que se apoiam numa multiplicidade de critérios não existindo em muitos casos uma definição oficial consideram-se como espaços ou meios não urbanos. As tipologias mais comuns baseiam-se fundamentalmente em critérios quantitativos - densidade populacional, dimensão dos lugares e emprego agrícola - sendo em alguns casos considerada a utilização dos solos e outros indicadores de natureza económica e social ${ }^{(2)}$.

Relativamente às populações dos centros urbanos e não urbanos existem diferenças culturais e sociais que influenciam os conhecimentos, atitudes e comportamentos de saúde relativas à SIDA. Estas diferenças são ainda acentuadas com a falta de recursos humanos na área da saúde. Em comparação com os países da União Europeia, Portugal é dos países que têm menos profissionais de saúde por habitante ${ }^{(3)}$.

A complexidade das questões que envolvem a SIDA, implica uma acção direccionada na vertente da prevenção, a fim de evitar a propagação da doença. O combate à SIDA é feito através da adopção e manutenção de comportamentos seguros. A mudança de comportamentos constitui um processo complexo, que se desenvolve em várias etapas e que difere de indivíduo para indivíduo de acordo com as suas características psicológicas, sociais e culturais ${ }^{(4-5)}$.
A proporção de casos diagnosticados em adultos jovens e adolescentes na transmissão heterossexual tem aumentado progressivamente. Está estimado que $1 / 4$ dos adolescentes irá ter doenças sexualmente transmissíveis antes de chegar à Universidade ${ }^{(6-7)}$.

É fundamental defender uma intervenção o mais precoce possível. Atendendo a que a adolescência é o período de transição entre a infância e a idade adulta e considerase que existe neste período uma plasticidade e vulnerabilidade da personalidade individual capaz de mudança estando mais susceptível para absorver conhecimentos e conceitos salutares que levarão os adolescentes a uma maturidade física, psicológica e sexual mais salutar no conceito lato da saúde ${ }^{(6)}$

Urge implementar estratégias e programas preventivos em idades o mais precoces possíveis, atendendo aos factores de risco envolvidos, pois é um problema de Saúde Pública e do próprio indivíduo. Contudo, o sucesso final destas medidas, depende também da vontade dos indivíduos assumirem e/ou manterem comportamentos de saúde adequados.

\section{OBJETIVO}

No âmbito desta investigação foi formulado o seguinte objectivo: Identificar e comparar conhecimentos, atitudes e comportamentos preventivos, sobre a SIDA em adolescentes que frequentam escola inserida em meio não urbano com os adolescentes que frequentam escola inserida em meio urbano.

\section{MÉTODO}

A investigação desenvolveu-se durante um período de três meses com a identificação dos conhecimentos, atitudes e comportamentos de 826 adolescentes com idades compreendidas entre os 15 e 18 anos de ambos os sexos, que frequentam o $10^{\circ}, 11^{\circ}$ e $12^{\circ}$ anos do ensino Secundário das Escolas Secundárias de Oliveira Frades e Vouzela que se encontram inseridas em meio não urbano e a Escola Secundária Emídio Navarro de Viseu que se encontra inserida em meio urbano. A razão porque foram seleccionadas duas escolas secundárias inseridas em meio não urbano, prendeuse com a necessidade de obter um número de participantes com algum significado (371 adolescentes).

\section{Hipótese de investigação}

Foi definida a hipótese: Há diferenças estatisticamente significativas entre os conhecimentos, atitudes e comportamentos sobre HIV/SIDA dos adolescentes que frequentam 
a escola inserida em meio não urbano daqueles que frequentam a escola inserida em meio urbano.

\section{Variáveis dependente e independente}

No nosso estudo propusemo-nos a investigar como variável dependente, os conhecimentos, atitudes e comportamentos dos adolescentes face ao HIV/SIDA.

Para se avaliar os conhecimentos dos adolescentes sobre SIDA de cada meio definiram-se as seguintes dimensões:

Quadro 1 - Dimensões de análise dos Conhecimentos dos Adolescentes sobre a SIDA-Coimbra - 2005

\begin{tabular}{|l|l|}
\hline Dimensão I & SIDA: uma doença normal \\
\hline Dimensão II & Percepção da SIDA como ameaça grave \\
\hline Dimensão III & Percepção da vulnerabilidade individual à SIDA \\
\hline Dimensão IV & Formas de transmissão da SIDA \\
\hline Dimensão V & Riscos de contágio da SIDA \\
\hline
\end{tabular}

Quadro 2 - Dimensões de análise das Atitudes e Comportamentos dos adolescentes face à SIDA - Coimbra - 2005

\begin{tabular}{|l|l|}
\hline Dimensão I & Comportamento sexual \\
\hline Dimensão II & Comportamentos adaptados face à SIDA \\
\hline Dimensão III & Atitudes face à SIDA \\
\hline
\end{tabular}

Os formatos de resposta são variados para as diferentes dimensões.

Como variáveis independentes foram consideradas as variáveis a seguir definidas sendo operacionalizadas em várias categorias: Idade (variável contínua), Sexo (masculino e feminino), Ano de escolaridade $\left(10^{\circ}, 11^{\circ}, 12^{\circ}\right.$ anos), Área de residência (meio urbano e meio não urbano).

\section{Instrumento de colheita de dados}

Para a colheita de dados, efectuou-se a aplicação de um questionário de preenchimento individual e anónimo, baseado no modelo conceptual HEALTH BELIEF MODEL, já validado em estudo à população portuguesa ${ }^{(8)}$. Foi estabelecido um contacto personalizado com todos os adolescentes a quem foram colhidos os dados, tendo sido explicado a finalidade da investigação, o carácter voluntário da participação, o compromisso de garantia de confidencialidade dos dados, a disponibilidade para o esclarecimento de dúvidas e pedida autorização para o seu preenchimento. Foi previsto o tempo de 10 minutos para o seu preenchimento.

\section{Tratamento estatístico}

O tratamento dos dados obtidos foi efectuado em computador, através do programa Excel 2000 e SPSS (Statistical Package and Social Science), utilizando a estatística inferencial e descritiva.
Para analisar a relação entre a variável dependente e as variáveis independentes efectuou-se a aplicação do teste do Qui-Quadrado, ao qual esteve associada uma probabilidade que foi utilizada por forma a aceitar ou rejeitar a hipótese formulada. A decisão de aceitação surgiu em função do valor da probabilidade em comparação com o valor da significância fixado. Se o valor da probabilidade fosse inferior ao valor do nível de significância ( $a ́=0.05$ ) a hipótese era aceite, se pelo contrário for superior a hipótese era rejeitada.

\section{População alvo e amostra de estudo}

A população alvo é constituída por alunos que frequentam o ensino secundário das Escolas Secundárias já identificadas, de ambos os sexos e com idades compreendidas entre os 15 e os 18 anos. Para atingir o objectivo proposto com a realização deste estudo a escolha mais ponderada apontou para uma amostra probabilística.

O estudo assenta na amostra de 826 adolescentes, dos quais $55,1 \%$ frequentam a escola inserida no meio urbano e $44,9 \%$ frequentam a escola inserida em meio não urbano. A idade média dos adolescentes era de 16,5 anos, sendo 58,3\% do sexo feminino. O número de inquiridos por ano de escolaridade variou entre $39,5 \%$ para o $11^{\circ}$ ano, $39 \%$ para o $10^{\circ}$ ano e $21,5 \%$ para o $12^{\circ}$ ano. 


\section{Caracterização das escolas do estudo}

Esta caracterização baseia-se numa abordagem inicial em termos de ranking e de projectos em parceria com a saúde.

Relativamente ao ranking das escolas portuguesas e em função das médias de todas as notas obtidas nos exames das oito disciplinas consideradas (Matemática, Português, Português A, Biologia, Física, Química, Psicologia e História) a classificação foi a seguinte e por ordem decrescente: Escola Secundária de Vouzela - 12 valores; Escola Secundária Emídio Navarro de Viseu - 11,2 valores; Escola Secundária de Oliveira de Frades - 10,4 valores.

Relativamente a projectos, são parceiros os Centros de Saúde das respectivas localidades e as escolas secundárias do nosso estudo no Programa de Promoção e Educação para a Saúde (PPES). Na Escola Secundária de Oliveira de Frades e no âmbito do programa PPES foi implementado o "gabinete do adolescente" cujo principal objectivo consiste na abordagem de comportamentos de risco na adolescência, como o uso de substâncias nocivas e o comportamento sexual, este gabinete funciona quinzenalmente das 14 às 17 horas com a presença da Enfermeira Especialista em Saúde Infantil e Pediátrica.

\section{APRESENTAÇÃOE DISCUSSÃO DE RESULTADOS}

Os resultados serão apresentados em dois momentos: o primeiro é referente à globalidade das respostas do questionário de todos os adolescentes envolvidos no estudo de uma forma resumida; o segundo é referente aos testes das hipóteses em função das dimensões traçadas.

\section{Primeiro momento}

Dos adolescentes estudados a média de idades era de 16,5 anos e 58,2\% eram do sexo feminino. A maioria deles frequentavam o $11^{\circ}$ ano $(39,5 \%)$ e o $10^{\circ}$ ano $(39 \%)$, os restantes $21,5 \%$ frequentavam o $12^{\circ}$ ano.

Em termos de identificação de conhecimentos, atitudes e comportamentos detectou-se que a grande maioria dos adolescentes reconhece a SIDA como uma doença normal (94\%), sendo um tanto possível que venha a afectar muita gente em Portugal (59\%) e evidenciando uma forte vulnerabilidade à SIDA. Das formas de transmissão da SIDA o item referido mais vezes $(81 \%)$ foi através de contacto sexual com pessoa infectada, seguida da via sexual $(72,6 \%)$ e através de seringa usada por outras pessoas e nos hospitais (65,6\%). Quanto aos riscos de contágio $80,9 \%$ dos adolescentes considera que uma pessoa corre muito perigo ao ter relações sexuais com pessoa infectada mesmo sem sintomas. As práticas sexuais com múltiplos parceiros diminuiria do período de 3 anos para 6 meses de $12 \%$ para $5,2 \%$ e aumentou ligeiramente com uma só pessoa de $12,6 \%$ para $14 \%$.
Referem ter adoptado comportamentos preventivos face à SIDA 52,5\% dos adolescentes e 42\% não os adoptaram, bem como a maioria dos adolescentes $59,5 \%$ não alteraram os seus hábitos e comportamentos sexuais em virtude da existência da SIDA. A maioria dos adolescentes $64,7 \%$ considera o preservativo um tanto seguro, e devem preocuparse em usar em todas as circunstâncias (94,8\%). A divulgação do passado sexual é aceite pela maioria dos adolescentes $(63,8 \%)$ e apenas $3,4 \%$ referem que não devem falar. A pesquisa de anticorpos da SIDA é admitida pela maioria dos adolescentes.

\section{Segundo momento}

\section{Conhecimentos}

\section{Dimensão I-SIDA: Uma doença normal}

Verificámos que 94,7\% dos adolescentes do meio urbano têm conhecimento da SIDA como podendo afectar qualquer pessoa enquanto que no meio não urbano esse valor é de $93 \%$. Apenas 5,3\% dos adolescentes do meio urbano e $7 \%$ do meio não urbano não têm conhecimento da SIDA como podendo afectar qualquer pessoa.

Para testar a hipótese "Há relação entre o meio de inserção dos adolescentes e o conhecimento da SIDA como uma doença normal" aplicámos o teste do Qui-Quadrado $\left(\mathrm{X}^{2}\right)$ ao nível de significância de 5\% e 1 grau de liberdade.

Obtivemos um valor de $\mathrm{X}_{\mathrm{p}}{ }^{2}=1,08\left(\mathrm{X}_{\mathrm{t}}^{2=} 3,84\right)$ cujo valor de correcção de continuidade é de 0,80 a que está associada uma probabilidade de $\mathrm{p}=0,299$, o que implica a rejeição da hipótese formulada. Portanto não se confirma estatisticamente nesta amostra que o meio de inserção influencia o conhecimento da SIDA como uma doença normal.

\section{Dimensão II - Percepção da SIDA como ameaça grave}

Verificámos que 70,1\% dos adolescentes do meio urbano não têm conhecimento da SIDA como uma doença grave, enquanto que no meio não urbano esse valor é ligeiramente inferior sendo de $62 \%$.

Para testar a hipótese " Há relação entre o meio de inserção dos adolescentes e o conhecimento da SIDA enquanto ameaça grave" aplicámos o teste do Qui-Quadrado $\left(\mathrm{X}_{2}\right)$ ao nível de significância de 5\% e 1 grau de liberdade.

Obtivemos um valor de $\mathrm{X}_{\mathrm{p}}^{2}=6,038\left(\mathrm{X}_{\mathrm{t}}^{2=} 3,84\right)$ cujo valor de correcção de continuidade é de 5,68 a que está associada uma probabilidade de $\mathrm{p}=0,014$, o que implica a aceitação da hipótese formulada, ou seja nesta população confirma-se estatisticamente que o meio de inserção influencia o conhecimento da SIDA enquanto ameaça grave.

Da análise dos resultados parece deduzir-se que os adolescentes não vêem a SIDA como uma ameaça grave. Um estudo $^{(8)}$ com uma população diferente obteve percentagens 
semelhantes. As pessoas adoptam comportamentos preventivos desde que sintam a doença como uma ameaça grave e se sintam vulneráveis a ela ${ }^{(7-10)}$. Parece haver relação entre 0 conhecimento da SIDA como ameaça grave e o meio de inserção dos adolescentes. As diferenças de opiniões encontradas entre os adolescentes que se encontram inseridos em meio urbano e não urbano parece-nos dever-se ao facto de ao nível escolar não existir a preocupação de esclarecer esta temática aos adolescentes do meio urbano, assim como a nível familiar, da saúde e comunidade. Permitindo, desta forma, um desconhecimento pouco saudável, que pode ter consequências catastróficas no futuro destes jovens, se pensarmos que é uma situação que nos rodeia.

\section{Dimensão III - Percepção da vulnerabilidade individual à SIDA}

Verificámos que quanto à percepção da vulnerabilidade individual à SIDA os adolescentes do meio urbano e meio não urbano apresentam valores próximos de conhecimento, respectivamente de $64 \%$ e $62 \%$.

Para testar a hipótese "Há relação entre o meio de inserção dos adolescentes e o conhecimento da percepção da vulnerabilidade individual à SIDA" aplicámos o teste do Qui-Quadrado $\left(\mathrm{X}^{2}\right)$ ao nível de significância de 5\% e 1 grau de liberdade.

Obtivemos um valor de $\mathrm{X}_{\mathrm{p}}^{2}=0,338\left(\mathrm{X}_{\mathrm{t}}^{2=} 3,84\right)$ cujo valor de correcção de continuidade é de 0,259 a que está associada uma probabilidade de $\mathrm{p}=0,561$, o que implica a rejeição da hipótese formulada, ou seja, não se confirma estatisticamente nesta amostra que o meio de inserção influencia o conhecimento da percepção da vulnerabilidade individual à SIDA.

Este sentimento de vulnerabilidade à doença constitui uma condição para adoptar comportamentos preventivos e a sua ausência pode constituir em si um factor favorável à difusão da mesma ${ }^{(8)}$.

\section{Dimensão IV - Conhecimento das formas de transmissão da SIDA}

Verificámos que $82,4 \%$ dos adolescentes do meio urbano têm conhecimentos modestos sobre as formas de transmissão da SIDA, sendo este valor de $86 \%$ para os adolescentes do meio não urbano. Nenhum do grupo dos adolescentes apresentam conhecimentos profundos sobre as formas de transmissão da SIDA.

Para testar a hipótese "Há relação entre o meio de inserção dos adolescentes e os conhecimentos das formas de transmissão da SIDA" aplicámos o teste do Qui-Quadrado $\left(\mathrm{X}^{2}\right)$ ao nível de significância de 5\% e 1 grau de liberdade.

Obtivemos um valor de $\mathrm{X}_{\mathrm{p}}^{2}=1,94\left(\mathrm{X}_{\mathrm{t}}^{2=} 3,84\right)$ cujo valor de correcção de continuidade é de 1,68 a que está associada uma probabilidade de $\mathrm{p}=0,164$, o que implica a rejeição da hipótese formulada, ou seja, não se confirma estatisticamente nesta amostra que o meio de inserção influencia os conhecimentos dos adolescentes acerca das formas de transmissão da SIDA.

\section{Dimensão V-Riscos de contágio da SIDA}

Verificámos que $58,7 \%$ dos adolescentes do meio urbano têm conhecimentos sobre os riscos de contágio de SIDA, sendo este valor de $49,6 \%$ para os adolescentes do meio não urbano. Não apresentam conhecimentos sobre os riscos de contágio da SIDA 38,5\% dos adolescentes do meio urbano e $34,5 \%$ para os adolescentes do meio não urbano. É de salientar que $15,9 \%$ dos adolescentes do meio não urbano têm conhecimentos profundos sobre os riscos de contágio da SIDA, sendo este valor de $2,9 \%$ para os adolescentes do meio urbano.

Para testar a hipótese "Há relação entre o meio de inserção dos adolescentes e os conhecimentos sobre os riscos de contágio em relação à SIDA “ aplicámos o teste do QuiQuadrado $\left(\mathrm{X}_{2}\right)$ ao nível de significância de 5\% e 1 grau de liberdade.

Obtivemos um valor de $\mathrm{X}_{\mathrm{p}}^{2}=43,87\left(\mathrm{X}_{\mathrm{t}}^{2=} 5,99\right)$ cujo valor de correcção de continuidade é de 5,68 a que está associada uma probabilidade de $\mathrm{p}=0,014$, o que implica a aceitação da hipótese formulada, ou seja nesta população confirma-se estatisticamente que o meio de inserção influencia os conhecimentos dos adolescentes em relação aos riscos de contágio da SIDA.

As diferenças de opinião entre os adolescentes que se encontram inseridos em meio urbano e meio não urbano parecem dever-se a deficiente informação no meio urbano acerca da SIDA, que pelos complexos problemas médicos, psicológicos, sociais e económicos, que arrasta consigo, despoleta a necessidade de uma prestação de cuidados extremamente alargada e que se envolvam várias instituições e até entidades ${ }^{(11)}$.

\section{Comportamentos e atitudes}

\section{Dimensão I - Comportamento sexual}

Verificámos que $80,6 \%$ dos adolescentes do meio não urbano alteraram os seus hábitos e comportamentos sexuais por causa da SIDA, sendo este valor de $78 \%$ para os adolescentes do meio urbano.

Do meio urbano $22 \%$ dos adolescentes não alteraram os seus hábitos e comportamentos sexuais por causa da SIDA, sendo este valor de $19,4 \%$ para os adolescentes do meio não urbano.

Para testar a hipótese “Há relação entre o meio de inserção dos adolescentes e a alteração de hábitos e comportamentos sexuais face à SIDA" aplicámos o teste do Qui-Quadrado $\left(\mathrm{X}^{2}\right)$ ao nível de significância de 5\% e 1 grau de liberdade. 
Obtivemos um valor de $\mathrm{X}_{\mathrm{p}}^{2}=0,82\left(\mathrm{X}_{\mathrm{t}}^{2=} 3,84\right)$ cujo valor de correcção de continuidade é de 0,67 a que está associada uma probabilidade de $\mathrm{p}=0,390$, o que implica a rejeição da hipótese formulada, ou seja, não se confirma estatisticamente nesta amostra que o meio de inserção influencia os comportamentos sexuais dos adolescentes em relação à SIDA.

Admitimos que se deva, provavelmente ao facto de haver adolescentes que ainda não tiveram qualquer experiência sexual.

\section{Dimensão II - Comportamentos adoptados face ao HIV/SIDA}

Verificámos que 74,4\% dos adolescentes do meio não urbano adoptam um comportamento de risco, sendo o valor de $71,2 \%$ para os adolescentes do meio urbano.

De salientar que $28,8 \%$ dos adolescentes do meio urbano têm um comportamento preventivo sendo este valor de 25,6\% para os adolescentes do meio não urbano.

Para testar a hipótese "Há relação entre o meio de inserção dos adolescentes e os comportamentos preventivos em relação à SIDA" aplicámos o teste do Qui-Quadrado $\left(\mathrm{X}^{2}\right)$ ao nível de significância de 5\% e 1 grau de liberdade.

Obtivemos um valor de $\mathrm{X}_{\mathrm{p}}^{2}=1,04\left(\mathrm{X}_{\mathrm{t}}^{2=} 3,84\right)$ cujo valor de correcção de continuidade é de 0,89 a que está associada uma probabilidade de $\mathrm{p}=0,307$, o que implica a rejeição da hipótese formulada, ou seja, não se confirma estatisticamente nesta amostra que o meio de inserção influencia os comportamentos preventivos em relação à SIDA.

As razões que se podem apontar e que provavelmente explicam este resultado são: dificuldades monetárias para a aquisição do preservativo; inibição própria destas idades; meio sócio cultural rígido e bastante restrito. Relembrando o modelo de crença, a adopção de comportamentos preventivos depende da facilidade na execução das acções recomendadas ${ }^{(11)}$.

\section{Dimensão III - Atitudes face à SIDA}

Verificámos que $90,6 \%$ dos adolescentes do meio urbano têm atitudes de risco, sendo este valor de $83,3 \%$ para os adolescentes do meio não urbano.

De salientar que $16,7 \%$ dos adolescentes do meio não urbano têm atitudes preventivas, sendo este valor de 9,5\% para os adolescentes do meio urbano.

Para testar a hipótese "Há relação entre o meio de inserção dos adolescentes e as atitudes preventivas em relação à SIDA" aplicámos o teste do Qui-Quadrado $\left(\mathrm{X}^{2}\right)$ ao nível de significância de 5\% e 1 grau de liberdade.

Obtivemos um valor de $X_{p}^{2}=9,71\left(X_{t}^{2=} 3,84\right)$ cujo valor de correcção de continuidade é de 9,07 a que está associada uma probabilidade de $p=0,002$, o que implica a aceitação da hipótese formulada, ou seja, nesta população confirmase estatisticamente que o meio de inserção influencia as atitudes preventivas em relação à SIDA.

Relativamente à atitude, a questão fulcral é que ela é complexa, isto é, simultaneamente estável e susceptível de mudar, tem algo de subjectivo e de objectivo, tem origem interna e externa. A atitude, não existe no abstracto, está ligada a crenças e valores, com os quais interage, determinando-se mutuamente ${ }^{(12)}$. As diferenças de opiniões entre os adolescentes do meio urbano e do meio não urbano revelam uma atitude mais adequada dos adolescentes inseridos no meio não urbano. Estes resultados vêm confirmar algumas diferenças que se verificaram entre os meios não urbano e urbano. Um dos factores que poderá estar na tese desta diferença poderá ser o facto de que no meio não urbano, as normas sociais são muitas vezes diferentes das existentes em meio urbano, e em que as crenças podem com maior facilidade ter uma influência positiva ao nível dos comportamentos e atitudes ${ }^{(12)}$. Vê-se, assim reforçada a ideia de que não é possível conceber o indivíduo fora do contexto no qual se insere, que se constitui enquanto uma matriz inter relacional determinante para o seu ser e para o seu estar.

\section{CONCLUSÃO}

Os conhecimentos dos adolescentes avaliados em cinco dimensões permite-nos concluir que: (1) o conhecimento da SIDA como podendo afectar qualquer pessoa é independente do meio de inserção dos adolescentes; (2) o conhecimento da SIDA como ameaça grave depende do meio de inserção dos adolescentes, sendo que os adolescentes inseridos em meio não urbano demonstram maior conhecimento da SIDA como ameaça grave; (3) o conhecimento da vulnerabilidade individual à SIDA é independente do meio de inserção dos adolescentes; (4) o conhecimento das formas de transmissão da SIDA é independente do meio de inserção dos adolescentes; (5) o conhecimento de riscos de contágio da SIDA depende do meio de inserção dos adolescentes, sendo que os adolescentes inseridos em meio não urbano demonstraram mais conhecimentos sobre os riscos de contágio da SIDA.

Os comportamentos e atitudes dos adolescentes face à SIDA avaliados em três dimensões permite-nos concluir que: (1) a alteração de hábitos e comportamentos sexuais face à SIDA é independente do meio de inserção dos adolescentes; (2) os comportamentos preventivos em relação à SIDA são independente do meio de inserção dos adolescentes; (3) o meio de inserção dos adolescentes influencia as atitudes preventivas em relação à SIDA, observando-se que a grande maioria dos adolescentes do meio urbano têm atitudes de risco e menores atitudes preventivas. 
Face aos resultados observados e da análise efectuada, é urgente uma maior contribuição de todos na prevenção da SIDA, proporcionando uma informação adequada e persis-

\section{REFERÊNCIAS}

1. Portugal. Instituto Nacional de Estatística. Indicadores estatísticos gerais. Lisboa; 2001.

2. Portugal. Instituto de Desenvolvimento Rural e Hidráulica. Definição e caracterização da zona abrangida. Lisboa; 2003. p. 7-10.

3. Cabral MV. Saúde e incapacidades. In: Portugal. Instituto Nacional de Estatística. Portugal social: 1991-2001. Lisboa; 2003. p. 9-10

4. Espanha. Ministério de Sanidade e Consumo. Epidemiologia y prevencion del Sida. Menorca; 1989.

5. Frasquilho MA. Comportamento e educação para a saúde: comportamento problema e comportamento saudável segundo os adolescentes. Rev Port Saúde Pública. 1998;16(1):19-31.

6. Lucas JS. Mudanças em percepções e atitudes favoráveis à prevenção da SIDA. Rev Port Saúde Pública. 1990;8(4):61-4.

7. Joint United Nations Programme on HIV/AIDS (UNAIDS). Adults and children estimated to be living with HIV/AIDS as of end 1999. Geneva; 2000. tente, que permita aos adolescentes optar por estilos de vida mais saudáveis.

8. Lucas JS. Os portugueses e a SIDA: inquérito nacional sobre conhecimentos, atitudes e comportamentos associados com a SIDA. Rev Port Saúde Pública. 1987;5(3/4):98-100.

9. Lucas JS. A sexualidade desprevenida dos portugueses. Lisboa: Mcgraw-Hill de Portugal; 1993.

10. Organização Mundial de Saúde (OMS). Compartilhar o desa fio HIV: um vírus que divide perigosamente. Rev Port Saúde Pública. 1992;9(4):23-6.

11. Almeida F. Adolescentes e a Sida: conhecimentos, atitudes e comportamentos associados com a Sida nos adolescentes no Concelho de Cantanhede. Lisboa: Escola Nacional de Saúde Pública; 1997.

12. Gomes A. As atitudes dos enfermeiros perante doença grave [dissertação]. Coimbra: Faculdade de Psicologia e Ciências da Educação, Universidade de Coimbra; 1993. 